\title{
Cardiovascular Physiological Effects Generated by Exercise with Restriction of Blood Flow: A Mini Review
}

\author{
Igor S Bonfim ${ }^{1,2^{*}}$ \\ ${ }^{1}$ Doctarate Student at Post Graduation Programme in Rehabilitation Sciences (UNISUAM), Brazil \\ ${ }^{2}$ Professor of the Graduate Course in Physiotherapy, Estácio de Sá University, UNESA, Brazil
}

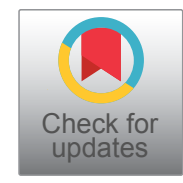

\begin{abstract}
Blood flow restriction exercise is a training method that partially restricts arterial flow and total venous flow in skeletal musculature during exercise. The study aims to demonstrate the physiological cardiovascular effects generated by exercise with blood flow restriction, as well as to point out evidence about the safety in the application of the method. Exercise with blood flow restriction is capable of causing beneficial physiological changes in the cardiovascular system and seems to be a safe method of application together with physical exercise, however standardization measures of the method need to be better studied with regard to individuals with cardiovascular changes.
\end{abstract}

\section{Introduction}

The performance of exercises with reduced blood flow obtained by restricting vascularization proximal to the muscle developed by Dr. Yoshiaki Sato in Japan, was initially known as "kaatsu training". Currently, the term used for this modality is "blood flow restriction" or BFR (abbreviation of the English term blood flow restriction) [1]. The term partial vascular occlusion has also been used by clinicians. Exercise with blood flow restriction is a training method that partially restricts arterial flow and total venous flow in skeletal muscles during exercise [1].

Exercise with restricted blood flow has been used in diverse populations with healthy and sick individuals. To perform the technique, a tourniquet cuff is required in the proximal regions of the limb, which, when inflated, generates a gradual mechanical vascular compression under the cuff, resulting in partial restriction of arterial and venous blood flow to structures distal to the cuff. This method is generally used for hypertrophy and increased muscle strength, however, the method is capable of generating systemic physiological effects through hypoxia generated in the muscle environment, which can be observed in addition to the musculoskeletal system $[1,2]$.

An ischemic and hypoxic muscle environment is generated during exercise with blood flow restriction to cause high levels of metabolic stress as well as mechanical strain when used in conjunction with exercise. Metabolic stress and mechanical stress are primary factors of hypertrophy and activate other mechanisms for inducing muscle growth. These proposed mechanisms include: Increased systemic hormonal production, cell edema, intramuscular anabolic/anti- catabolic signaling, and increased recruitment of fast-twitch fibers. However, blood flow restriction also demonstrates cardiovascular changes in physiology and consequently benefits in populations with cardiovascular pathologies [2].

The aim of this review is to demonstrate the physiological cardiovascular effects generated by exercise with blood flow restriction, as well as to point out evidence about the safety in the application of the method with regard to possible vascular damage.

\section{Theoretical Reference}

\section{Cardiovascular responses}

Centraland peripheralvascular responses are accompanied by increased oxygen demand in active skeletal muscles during exercise. At the systemic level, reductions in systolic and diastolic blood pressure were observed after using the technique. However, in the study by Jeffries, et al. no changes in blood pressure, resting heart rate or autonomic function were found [3]. There are divergences in the literature, with several studies reporting on change in pressure suggesting that more evidence is needed to understand the systemic

*Corresponding author: Igor S. Bonfim, Post Graduation Programme in Rehabilitation Sciences (UNISUAM), Rio de Janeiro, Brazil

Accepted: October 18, 2021

Published online: October 20, 2021

Citation: Bonfim IS (2021) Cardiovascular Physiological Effects Generated by Exercise with Restriction of Blood Flow: A Mini Review. Arch Addict Rehabil 3(1):119-121 
effects of exercise with blood flow restriction. The effect of exercise with blood flow restriction on the cardiovascular center depends on the level of flow restriction, exercise mode and application mode. Cuff removal during rest intervals, as happened in the study by Jeffries, et al. seems to reduce cardiovascular differences between exercise with blood flow restriction and conventional exercise [3].

\section{Peripheral vascular response and endothelial function}

Exercise with blood flow restriction has been shown to affect arterial compliance and endothelial function. Exercise with restricted blood flow causes vascular adaptations, including an up-regulation of transcription of genes involved in signaling pathways that contribute to exercise-induced angiogenesis. Specifically, increases in vascular endothelial mRNA expression, vascular endothelial growth factor (VEGF), vascular endothelial growth factor receptor (also known as KDR or VEGFR-2), hypoxia-inducible factor 1 alpha (HIF-1a), oxide isoforms nitric synthase (NOS) and serum VEGF [4].

In some randomized clinical trials, exercise with restricted flow increased the compliance of large arteries, suggesting a transient improvement in endothelial function $[5,6]$. The endothelium plays an important role in maintaining vascular homeostasis and regulating blood vessel function. Endothelial function in exercise studies with blood flow restriction is generally assessed by vascular reactivity, stimulating endothelial cells and measuring the degree of vasodilation in diameter and flow $[1,4]$. After exercise and blood flow release, reactive hyperemia occurs, translated as an increase in blood flow mediated by endothelial vasodilators. Increased blood flow represents a reduction in peripheral vascular resistance and can lead to a reduction in blood pressure. This has been verified in studies where hypotension was observed after resistance exercise with a sequential increase in blood flow [7].

\section{Safety in applying the method}

With the advent of the method and as it began to be used clinically, doubts regarding possible risks were raised by researchers. One of the main risks is the emergence of episodes of venous thromboembolism. A thrombus large enough to block blood flow, especially if located in smaller vessels, can result in local tissue ischemia and subsequent tissue death. Studies evaluating the interaction between vascular occlusion and thrombus appearance have not reported clinically relevant episodes. Tests to identify this relationship have used prothrombin fragment markers and the thrombin-antithrombin III test to assess the increase in thrombin generation immediately after training and found no significant increase $[1,5]$.

In addition, C-reactive protein, a protein that has been associated with clot formation, was also evaluated in the study and no significantly elevated levels were found. It is worth mentioning that samples from healthy individuals and those with heart disease were analyzed in the short term. However, some studies have also evaluated the association between the method and long-term thrombus formation and also did not find an increased risk for this complication $[1,5]$. An intriguing fact is that short periods of stasis can produce thrombus formation, although without adverse events. One mechanism to control the advancement of thrombus formation is through stimulation of the fibrinolytic system, and resistance training has demonstrated the ability to regulate the fibrinolytic pathway by stimulating the fibrinolytic system, increasing tissue plasminogen activator (tPA, a thrombus degrading protein in epithelial cell) [1].

Another point that deserves to be highlighted is the use of exercise with blood flow restriction in the population with hypertension. Effects on the hemodynamic system, such as increased systolic and diastolic blood pressure, were observed in patients with hypertension. It can be attributed to the accumulation of metabolites (lactate, protons, $\mathrm{K}^{+}$) and muscle metaboreflex, which results in increased sympathetic activity and increased blood pressure and heart rate [6]. The decrease in oxygen supply associated with muscle contraction is also a factor that provides a high ambient metabolic rate, which increases the afferent activity of the pressure reflex exercise, causing an increase in sympathetic nervous activity and, consequently, in blood pressure. Hypertensive individuals show increased sympathetic nervous activity, even at rest. These individuals also have dysfunction of baroreceptors and mechanoreceptors, the main mechanisms for regulating cardiovascular function during exercise $[4,6]$.

When associated with the maladjustment of these two mechanisms, exercise with blood flow restriction can promote exacerbated sympathetic nervous activity and consequent increase in blood pressure, even with the use of low loads during exercise. However, as mentioned above, a systematic review showed that exercise with blood flow restriction promotes a reduction in peripheral vascular resistance and, after exercise, a reduction in blood pressure, supporting the application of this type of exercise in hypertensive individuals cautious. The method prescription must take into account the individual clinical variables also used in the decision-making process for the prescription of exercises that do not use blood flow restriction for hypertensive patients $[2,4,7]$.

\section{Conclusion}

The literature on exercise with blood flow restriction in populations with cardiovascular alterations is emerging. Several questions about efficacy superior to exercise without restriction and contraindications need to be investigated. However, the literature demonstrates that the method of restricting blood flow during exercise can be applied safely and cautiously in this type of population. Standardization measures on cuff occlusion pressure, timing and revascularization need to be further studied with regard to individuals with cardiovascular alterations.

\section{Conflict of Interest Statement}

The author declares no conflict of interest.

\section{Acknowledgment}

This study was supported by Coordenação de Aperfeiçoamento de Pessoal de Nível Superior (CAPES, Brazil). 


\section{References}

1. Patterson SD, Hughes L, Warmington S, et al. (2019) Blood flow restriction exercise: Considerations of methodology, application, and safety. Front Physiol 10: 533.

2. Hughes L, Paton B, Rosenblatt B, et al. (2017) Blood flow restriction training in clinical musculoskeletal rehabilitation: $A$ systematic review and meta-analysis. Br J Sports Med 51: 10031011.

3. Jeffries $\mathrm{O}$, Waldron M, Pattison JR, et al. (2018) Enhanced local skeletal muscle oxidative capacity and microvascular blood flow following 7-day ischemic preconditioning in healthy humans. Front Physiol 9: 463.
4. DA CUNHA NASCIMENTO Dahan, SCHOENFELD Brad J, PRESTES Jonato (2020) Potential implications of blood flow restriction exercise on vascular health: A Brief Review. Sports Med 50: 7381.

5. Tennent DJ, Hylden CM, Johnson AE, et al. (2017) Blood flow restriction training after knee arthroscopy: A randomized controlled pilot study. Clin J Sport Med 27: 245-252.

6. Cuyul-vásquez I, Leiva-Sepúlveda A, Catalán-Medalla O, et al. (2020) Blood flow restriction training for people with cardiovascular disease: An exploratory review. Rehabilitacion(Madr) 54: 116-127.

7. Domingos E, Polito MD (2018) Blood pressure response between resistance exercise with and without blood flow restriction: A systematic review and meta-analysis. Life Sci 209: 122-131. 